\title{
The glycine brace: a component of Rab, Rho, and Ran GTPases associated with hinge regions of guanine- and phosphate-binding loops
} Andrew F Neuwald

Address: Institute for Genome Sciences and Department of Biochemistry \& Molecular Biology, University of Maryland School of Medicine, 801 West Baltimore St., BioPark II, Room 617, Baltimore, MD 21201, USA

Email: Andrew F Neuwald - aneuwald@som.umaryland.edu

Published: 5 March 2009

BMC Structural Biology 2009, 9:I I doi:10.1 I86/I472-6807-9-I I
Received: 24 October 2008

Accepted: 5 March 2009

This article is available from: http://www.biomedcentral.com/I472-6807/9/I I

(C) 2009 Neuwald; licensee BioMed Central Ltd.

This is an Open Access article distributed under the terms of the Creative Commons Attribution License (http://creativecommons.org/licenses/by/2.0), which permits unrestricted use, distribution, and reproduction in any medium, provided the original work is properly cited.

\begin{abstract}
Background: Ras-like GTPases function as on-off switches in intracellular signalling pathways and include the Rab, Rho/Rac, Ran, Ras, Arf, Sar and G $\alpha$ families. How these families have evolutionarily diverged from each other at the sequence level provides clues to underlying mechanisms associated with their functional specialization.
\end{abstract}

Results: Bayesian analysis of divergent patterns within a multiple alignment of Ras-like GTPase sequences identifies a structural component, termed here the glycine brace, as the feature that most distinguishes Rab, Rho/Rac, Ran and (to some degree) Ras family GTPases from other Raslike GTPases. The glycine brace consists of four residues: An aromatic residue that forms a stabilizing $\mathrm{CH}-\pi$ interaction with a conserved glycine at the start of the guanine-binding loop; a second aromatic residue, which is nearly always a tryptophan, that likewise forms stabilizing $\mathrm{CH}-\pi$ and $\mathrm{NH}-\pi$ interactions with a glycine at the start of the phosphate-binding P-loop; and two other residues (typically an aspartate and a serine or threonine) that, together with a conserved buried water molecule, form a network of interactions connecting the two aromatic residues.

Conclusion: It is proposed that the two glycine residues function as hinges and that the glycine brace influences guanine nucleotide binding and release by interacting with these hinges.

\section{Background}

Rab [1], Rho/Rac [2] and Ran [3,4] GTPases regulate diverse cellular processes including vesicle trafficking, cytoskeletal dynamics, cell polarity, membrane fusion, chromosome segregation, and nuclear transport. These proteins are a subgroup of the extended Ras-like superfamily of GTPases [5] (termed here the Ras-like GTPases), which function as signaling pathway on-off switches and which also include Arf, Arf-like (Arl), and Sar GTPases and $\alpha$ subunits of heterotrimeric $G$ proteins. Given an appro- priate upstream signal, Ras-like GTPases are turned 'on' by binding to GTP, resulting in their association with various 'effectors' that propagate the incoming signal to downstream components. Guanine nucleotide exchange factors (GEFs) facilitate this process by mediating the exchange of GTP for GDP. Ras-like GTPases are turned off upon hydrolysis of GTP to GDP, which results in termination of the signal and a shutting off of the pathway. GTPase activating proteins (GAPs) facilitate this process by stimulating the inherent GTPase activity of Ras-like GTPases. 
Ras-like GTPases are a subgroup of the phosphate-binding loop (P-loop) GTPases, which bind GTP via amino acid residues corresponding to several conserved motifs [6]. Two of these motifs are relevant to the analysis here: the Walker A (G-x $\left.\mathrm{x}_{4}-\mathrm{G}-\mathrm{K}-[\mathrm{ST}]\right)$ motif [7], which corresponds to the P-loop [8], and a [NT]-K-x-D motif, which occurs within the guanine-binding loop. The residues of the Walker A motif bind to the phosphate groups of GDP or GTP, whereas the residues of the guanine-binding motif bind to the guanine base and link it to the P-loop $[9,10]$. Here I identify a structural component, termed the glycine brace, that is specifically conserved in Rab, Ran and Rho/ Rac GTPases and that spans these two guanine nucleotide binding regions (Figure 1A).

\section{Results \\ Analysis of Ras-like GTPases}

Using a 'Bayesian partitioning with pattern selection' (BPPS) procedure [11], a multiple alignment of 91,406 Ploop GTPase sequences (the most conserved features of which are shown in Figure 2A) was optimally partitioned into two major subgroups - Ras-like GTPases versus other P loop GTPases - based on amino acid differences at evolutionarily-divergent residue positions. This identified about a dozen residue positions that are conserved in Raslike GTPases but not in other P-loop GTPases and that thus presumably reflect characteristic features shared by these on-off switches. Figure 1B highlights one of these conserved residues that is relevant to the analysis here namely a glycine or alanine immediately preceding the guanine-binding loop; the other residues will be described elsewhere (Neuwald, unpublished).

The alignment in Figure 1B, which is termed a 'contrast alignment', corresponds to the output produced by the BPPS procedure (although with only a short region relevant to this analysis shown here). As described in Figure $1 \mathrm{C}$, a contrast alignment consists of two contrasting subalignments, one of which contains strikingly conserved patterns that are non-conserved in the other sub-alignment. Thus the highlighted glycine or alanine in Figure 1B is specifically conserved within Ras-like GTPases, implying that it performs a function specific to these proteins. The analysis here provides some clues regarding this function - at least for those Ras-like GTPases that typically conserve a glycine at this position.

\section{Rab-related GTPases}

Application of the BPPS procedure $[11,12]$ to a sub-alignment consisting of Ras-like GTPases (an abridged version of which is shown in Figure 1B) resulted in a natural partitioning into two major Ras-like GTPase subgroups: Rab, Ran, Rac/Rho and certain Ras GTPases (the Rab-related subgroup) versus other Ras-like GTPases. The corresponding contrast alignment is shown in Figure 2B. The associ- ated pattern corresponds to six conserved residue positions that can be split into two groups: (i) the two adjacent residue positions (a threonine and an alanine) located between the Walker $\mathrm{B}$ aspartate and the glycine at the start of the switch II region; and (ii) four residue positions that structurally correspond to the glycine brace (Figure 3 ). The alanine residue within the first group is proposed to play a role in nucleotide exchange by pushing out the $\mathrm{Mg}++$ ion that coordinates with the guanine nucleotide phosphates [13].

The four pattern residues corresponding to the glycine brace include: (i) a conserved acidic or amidic residue ([DENQ]) immediately following the conserved glycine at the start of the P-loop (Asp19-Rab11A in Figures 2B and $3 \mathrm{~A})$; (ii) a tyrosine or phenylalanine ([YF]) (Tyr91-Rab11) within the $\beta 4$-strand, which forms a $\beta$-sheet with the $\beta$ strand directly preceding the guanine-binding loop; (iii) a serine or threonine ([ST]) (Thr98-Rab11) within the a3helix; and (iv) a tryptophan ([W])(or, rarely, a tyrosine or phenylalanine) (Trp105-Rab11A) also within the $\alpha 3$ helix. Rab, Ran and Rho GTPases generally conserve all of these patterns, but members of the Ras family typically lack matches to the canonical pattern at one or two positions and thus appear to have undergone additional evolutionary divergence. Despite these divergent features, however, by and large the Ras-family is still classified by the BPPS procedure into the Rab-related subgroup.

\section{The glycine brace}

The analysis in Figure 2B indicates that the most distinctive feature of Rab-related GTPases is the glycine brace (Figure 3), which is structurally characterized by nearly a dozen conserved atomic interactions. One of these is a $\mathrm{CH}-\pi$ interaction [14] between the aromatic residue corresponding to the [FY] pattern (Phe91-Rab11A in Figure $3 \mathrm{~A}$ ) and the conserved glycine (Gly123-Rab11A in Figures $1 \mathrm{~B}$ and $3 \mathrm{~A}$ ) within the adjacent parallel $\beta$-strand that immediately precedes the guanine-binding loop. The presence of glycine intrinsically destabilizes $\beta$ sheets, but this sort of aromatic-glycine interaction has been proposed to counteract this effect [15]. Thus, within Rabrelated GTPases, this $\mathrm{CH}-\pi$ interaction could stabilize the region directly preceding the guanine-binding loop, which conserves residues (Figure 2A) that bind both to the guanine base and to the P-loop (Figure 3).

Likewise, the tryptophan residue of the glycine brace (Trp105-Rab11A) often forms both a NH- $\pi$ [16] and a $\mathrm{CH}-\pi$ interaction with main-chain atoms of a glycine that is located at the start of the P-loop (Gly18-Rab11A in Figures $2 \mathrm{~A}$ and $3 \mathrm{~A}$ ) and that is highly conserved in P-loop GTPases (about $90 \%$ of the sequences in Figure 2A) and very highly conserved within glycine-brace GTPases (over $99 \%$ of the foreground sequences in Figure 2B). 

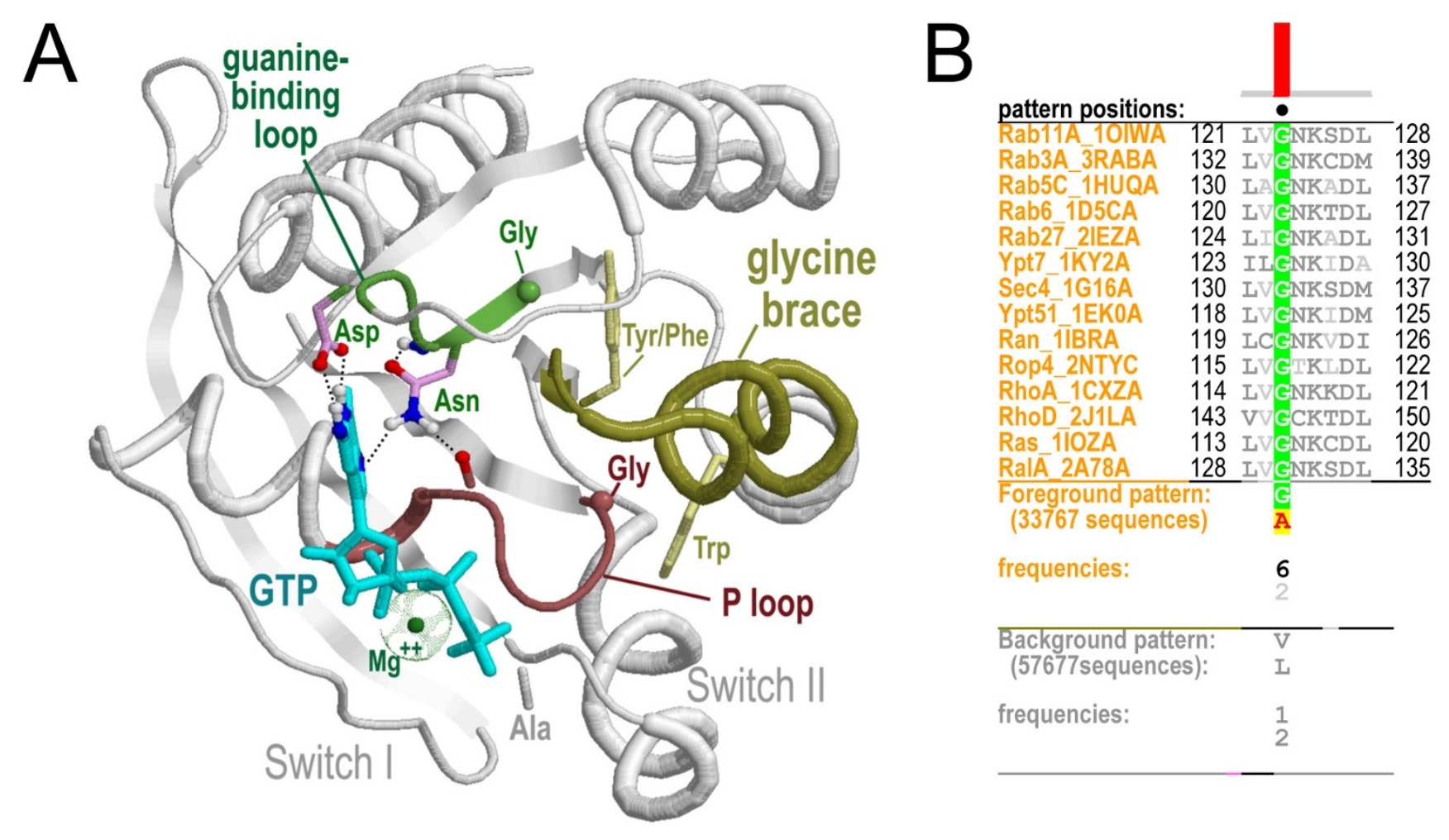

C

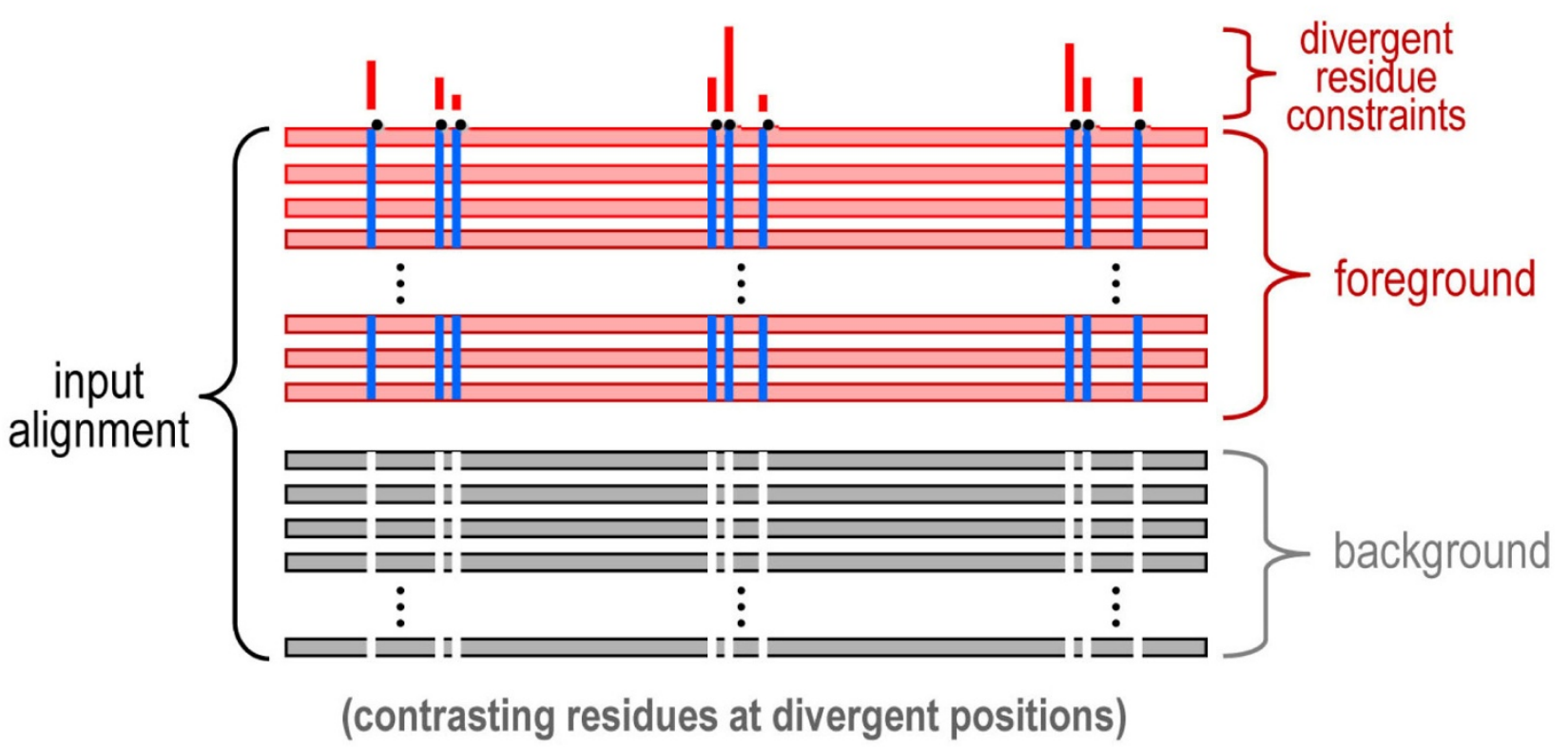

Figure I (see legend on next page) 
Figure I (see previous page)

Attributes and analysis of Rab-related and Ras-like GTPases. (A) Relevant structural features of Rab-related GTPases. The structure shown is that of Sec4 bound to a GTP analogue (pdb_id: Igl7)[34]. The two glycine residues proposed to function as hinges are indicated (with their $\alpha$-carbon atoms displayed as spheres). Also indicated are: a conserved alanine residue, repositioning of which is proposed to facilitate nucleotide exchange by occluding the $\mathrm{Mg}^{++}$binding site [I3]; the conserved asparagine and aspartate residues of the guanine-binding loop; and the conserved aromatic residues of the glycine brace. (B) A contrast alignment (defined in (C)) highlighting a conserved residue position near the guanine-binding loop that (along with other conserved residues outside of the region shown) distinguishes all Ras-like GTPases (the 'foreground') from other P-loop GTPases (the 'background'). Relevant foreground and background consensus residues at this position, along with corresponding (weighted) residue frequencies, are shown directly below the alignment of representative GTPase sequences (protein names and pdb identifiers are indicated in the leftmost column). Residue frequencies are given in integer tenths where, for example, a ' 6 ' indicates that the corresponding residue occurs in $60 \%-70 \%$ of the sequences. (C) Schematic representation of a contrast alignment [I2,3I]. A contrast alignment is the output returned by the CHAIN program's [I2] BPPS procedure [I I], which optimally partitions an input alignment into 'foreground' and 'background' sub-alignments such that the foreground sequences (red horizontal bars) strikingly conserve a pattern that is non-conserved in (or that contrasts with) the background sequences (gray horizontal bars) at pattern positions (blue vertical bars). The red vertical bars over pattern positions quantify the selective pressure (as previously defined [II]) that is imposed on the foreground relative to the background.

Two other glycine brace residues, an acidic or amidic residue (Asp19-Rab11A in Figures 2B and 3A) and a serine or threonine residue (T98-Rab11A), can participate in a network of hydrogen bonds linking the two aromatic residues associated with the P-loop and with the guaninebinding-loop. A buried water molecule, which is conserved across nearly all Rab-related GTPase crystal structures, also participates in this interaction network (Figure 3). In contrast, other Ras-like GTPases (i.e., members of the Arf, Arl, Sar and G $\alpha$ families) are characterized by a strikingly different network of interactions (unpublished observations).

\section{Non-glycine residues preceding the guanine-binding loop} Ninety-five percent of the sequences classified in this analysis as glycine-brace-containing GTPases (Figure 2B) harbor a glycine residue immediately preceding the guaninebinding loop. Many of the remaining glycine brace GTPases (3.7\%) harbor an alanine instead of a glycine at this position, whereas the rest $(1.4 \%)$ harbor some other residue. These non-glycine variants still conserve the fourresidue pattern associated with the glycine brace, and for variants of known structure the glycine brace phenylalanine or tyrosine still forms a $\mathrm{CH}-\pi$ interaction with the backbone $\alpha$-carbon hydrogen atom just as for typical glycine brace GTPases. These include three alanine variants: human RhoB (pdb_id: 2fv8)(Structural Genomics Consortium)(SGC), mouse M-ras; (pdb_id: 1x1r) [17], and Rab5a from Plasmodium falciparum (pdb_id: $\underline{3 c l v}$ )(SGC). This also includes one glutamine variant: mouse Rab23 (pdb_ids: 1z22, 1z2a) [18]. Often non-glycine substitutions at this position are conserved across an entire subfamily whose members span distinct phyla. For example, an alanine substitution is conserved across the Rab32 subfamily [19] whose members span at least eight phyla.
Thus such (relatively rare) substitutions appear to perform a functional role specific to these subfamilies.

\section{Discussion}

Because the glycine brace is the single structural feature that most distinguishes Rab-related GTPases from other Ras-like GTPases (Figure 2B), it presumably plays a critical functional role somehow related to the conserved atomic interactions described above. Given that the guaninebinding loop and the P-loop bind to both ends of GTP or GDP, the glycine brace could promote guanine nucleotide binding by stabilizing the conformations of these glycines, which could serve as hinges for opening and closing of these loops. Conversely, disruption of these aromaticglycine interactions could promote the release of GDP during nucleotide exchange. It is worthwhile noting in this context that the most buried residue $\left(\begin{array}{ll}164 & 2\end{array}\right)$ of Ran GTPase upon binding to its nucleotide exchange factor, RCC1 [20], is a lysine that is located near the center of the glycine brace $\alpha$ helix (Lys99-Ran in Figure 3D). Moreover, in the Ran-RCC1 crystal structure this lysine is inserted into the central hole of RCC1's $\beta$-propeller domain whereas the $\mathrm{CH}-\pi$ and $\mathrm{NH}-\pi$ interactions between the conserved tryptophan and the P-loop glycine are disrupted (compare Trp104-Ran in Figures 3C and 3D); taken together, this suggests a possible role for the glycine brace in nucleotide exchange within Ran GTPases.

What role might the non-glycine substitutions preceding the guanine-binding loop perform? To address this question, it should be noted that alanine is much more likely to occur as a substitute for glycine at this position than are other residues; this can be explained by the fact that both glycine and alanine promote structural flexibility [21-23]. However, as indicated by their Ramachandran plots, alanine is less flexible than glycine, suggesting that an 
A P-loop GTPases vs other proteins

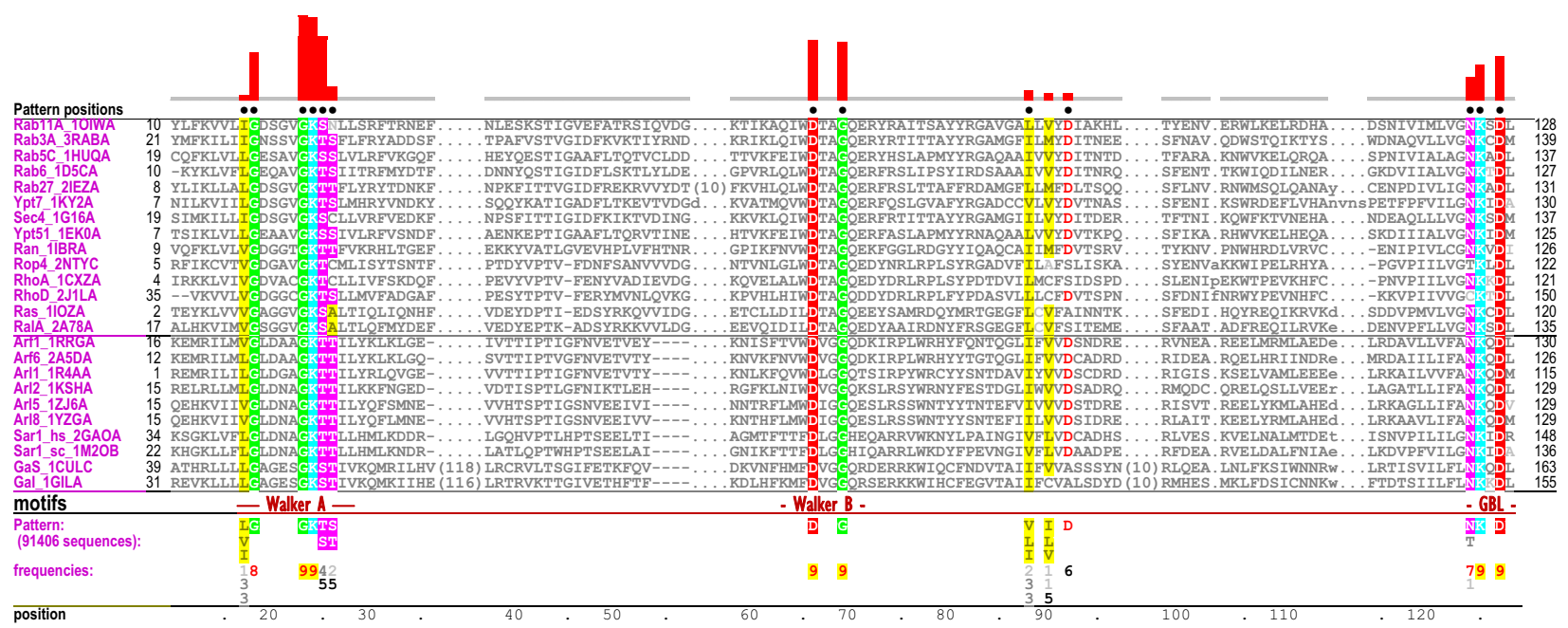

B Rab-related vs other Ras-like GTPases

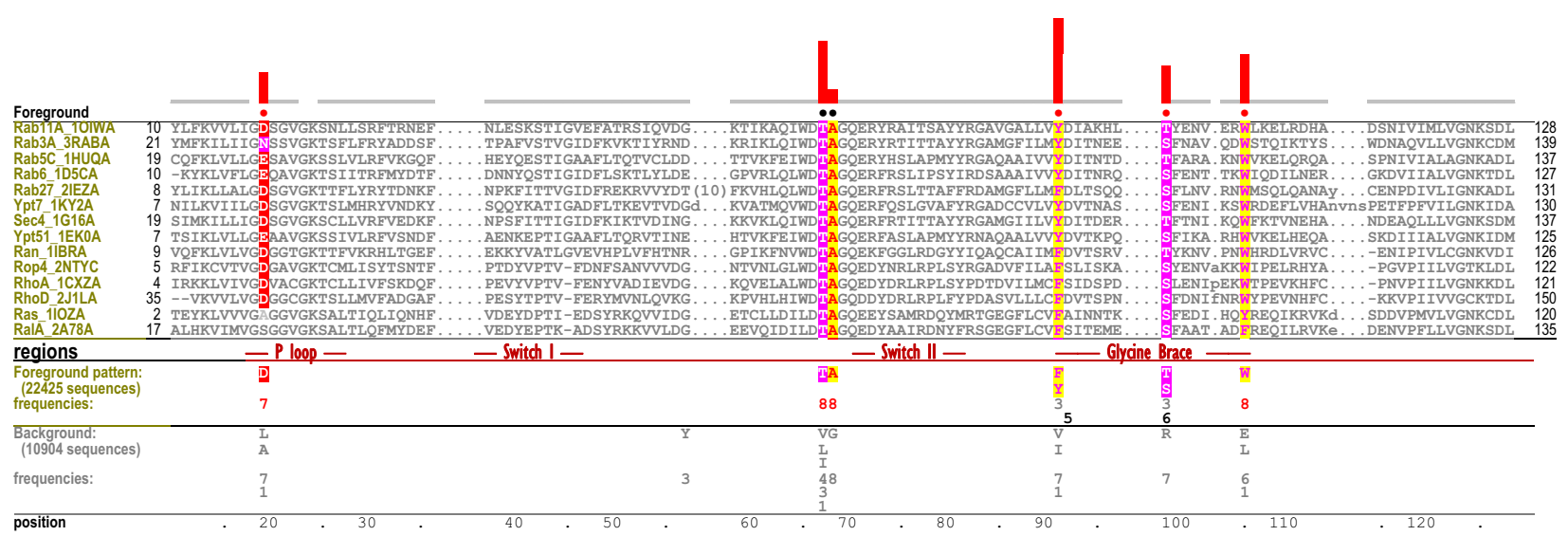

Figure 2

Contrast alignments showing the most distinctive sequence features of P-loop GTPases and of Rab-related GTPases. See legends to Figures IB,C for descriptions. (A) Distinctive sequence features of P-loop GTPases. The acronym 'GBL' stands for guanine-binding loop. (B) Distinctive sequence features of Rab-related GTPases. The foreground (Rab-related GTPases) includes Rab, Ran, Rho and some (but not all) Ras family GTPases; the background consists of other Ras-like GTPases.

alanine substitution decreases somewhat the flexibility of the guanine-binding loop. Perhaps turning on these alanine-variant GTPase switches at inappropriate times is highly detrimental, and, as a result, nucleotide exchange is suppressed (relative to other GTPase switches) by having a less flexible guanine-binding loop. Similarly, a non-glycine, non-alanine substitution seems likely to decrease the flexibility of the guanine-binding loop more dramatically; in these cases, the participation of specific exchange factor interactions may be required for nucleotide release leading to even more stringent, pathway-specific regulation.
Co-conservation of the glycine brace with the threonine and alanine of the Walker B (DTAG) motif (Figure 2) also is consistent with a role for the glycine brace in nucleotide exchange. Repositioning of the alanine is proposed to facilitate nucleotide exchange by occluding the $\mathrm{Mg++}$ binding site, leading to expulsion of the phosphate-associated $\mathrm{Mg}++$ ion [13]. Co-conservation of the glycine brace with this alanine thus suggests the possibility that all six of the Rab-related residues highlighted in Figure 2B somehow function as a unit to regulate nucleotide binding and release. 
A
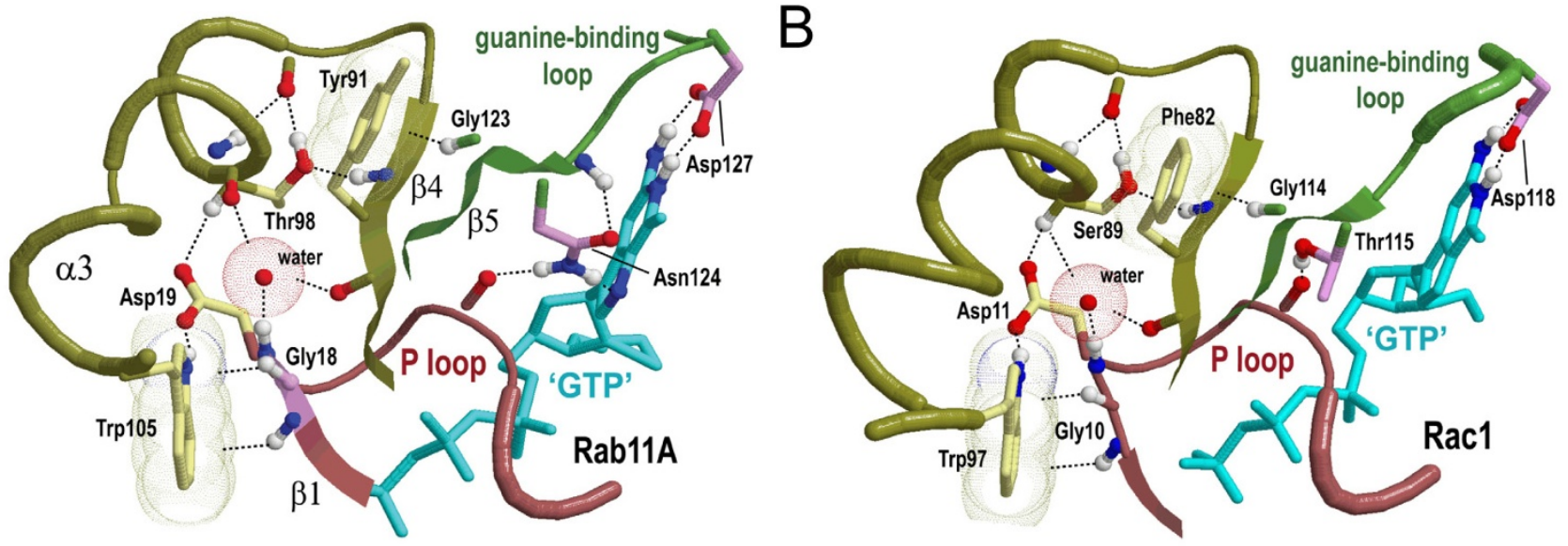

C
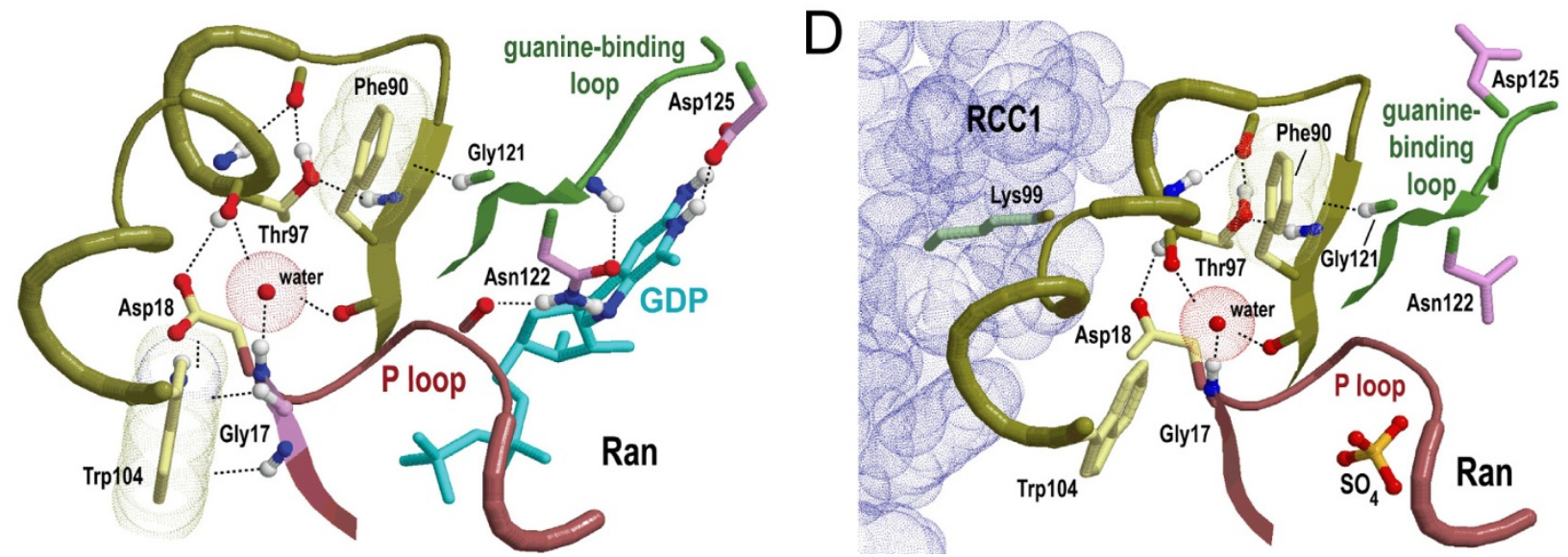

Figure 3

The glycine brace within crystal structures of representative Rab-related GTPases. Color scheme: backbone of the P-loop region, dark red; backbone of the glycine brace region, dark yellow; backbone of the guanine-binding loop region, green; side chains of residues characteristic of P-loop GTPases, magenta; side chains of residues characteristic of the Rab-related GTPases, yellow. (A) The Rab-family GTPase Rab I IA bound to a GTP analog (GTP $\gamma$ S) (pdb_id: loiw) [35]. (B) The Rho/Rac family GTPase Racl bound to a GTP analog (pdb_id: Ii4t) [36]. (C) Ran GTPase bound to GDP (pdb_id: Ibyu) [37]. (D) Ran GTPase bound to its exchange factor RCCl (pdb_id: $\underline{\lfloor 2 \mathrm{~m}}$ ) [20].

\section{Conclusion}

It is proposed that the two glycine residues, one preceding the guanine-binding loop and another preceding the Ploop, function as hinges and that the glycine brace influences guanine nucleotide binding or release by interacting with these hinges. This has obvious implications regarding the regulation of Rab-related GTPase switches via guanine nucleotide exchange. Of course, the precise manner in which the glycine brace might play a role in nucleotide exchange remains to be determined.

\section{Methods}

$P$ loop GTPases sequences were identified within the NCBI nr, env_nr and translated EST databases using PSIBLAST [24] and motif-based [25] search procedures. These sequences were multiply aligned using a variety of methods, including: manual curation of PSI-BLAST checkpoint files in conjunction with the PSI-BLAST alignment algorithm, MUSCLE $[26,27]$, Bayesian sequence alignment methods $[25,28,29]$, and the CE structurally-based alignment method [30]. Manual curation was performed in conjunction with structural analysis of sequence patterns using the CHAIN program [12]. Aligned sequences were partitioned into functionally divergent subgroups using a Bayesian partitioning with pattern selection (BPPS) procedure [11]; this identified both the Ras-like (Figure 1B) and Rab-related (Figure 2B) subgroups. The BPPS procedure is implemented within the CHAIN program [12]; for a review of CHAIN analysis see [31]. The Reduce program [32] was used to add hydrogen atoms to structural coordinate files. Molecular images were created by applying the Rasmol program [33] to the following 
structural coordinate files (pdb identifiers): 1g17[34], $\underline{10 i w}[35], \underline{1 i 4 t}[36], \underline{1 b y u}[37]$, and $\underline{1 \mathrm{i} 2 \mathrm{~m}}[20]$.

\section{Acknowledgements}

I thank Zhong Guo and an anonymous referee for helpful comments and suggestions. This work was funded by NIH Division of General Medicine Grant GM07854I.

\section{References}

I. Grosshans BL, Ortiz D, Novick P: Rabs and their effectors: achieving specificity in membrane traffic. Proc Natl Acad Sci USA 2006, I03(32): ||82|-| | 827.

2. Bustelo XR, Sauzeau V, Berenjeno IM: GTP-binding proteins of the Rho/Rac family: regulation, effectors and functions in vivo. Bioessays 2007, 29(4):356-370.

3. Clarke PR, Zhang C: Spatial and temporal coordination of mitosis by Ran GTPase. Nat Rev Mol Cell Biol 2008, 9(6):464-477.

4. Dasso M: The Ran GTPase: theme and variations. Curr Biol 2002, I 2(14):R502-508.

5. Leipe DD, Wolf YI, Koonin EV, Aravind L: Classification and evolution of P-loop GTPases and related ATPases. J Mol Biol 2002, 3I7(I):4I-72.

6. Wittinghofer A: The Functioning of Molecular Switches in Three Dimensions. In GTPases Edited by: Hall A. Oxford: Oxford University Press; 2000:244-310.

7. Walker JE, Saraste M, Runswick MJ, Gay NJ: Distantly related sequences in the alpha- and beta-subunits of ATP synthase, myosin, kinases and other ATP-requiring enzymes and a common nucleotide binding fold. Embo J I982, I(8):945-95I.

8. Saraste M, Sibbald PR, Wittinghofer A: The P-loop - a common motif in ATP- and GTP-binding proteins. Trends Biochem Sci 1990, I 5:430-434.

9. Pai EF, Kabsch W, Krengel U, Holmes KC, John J, Wittinghofer A: Structure of the guanine-nucleotide-binding domain of the Ha-ras oncogene product $\mathrm{p} 2 \mathrm{I}$ in the triphosphate conformation. Nature 1989, 34 I(6239):209-2I4.

10. Pai EF, Krengel U, Petsko GA, Goody RS, Kabsch W, Wittinghofer A: Refined crystal structure of the triphosphate conformation of $\mathrm{H}$-ras $\mathrm{p} 2 \mathrm{I}$ at $\mathrm{I} .35 \mathrm{~A}$ resolution: implications for the mechanism of GTP hydrolysis. EMBO | 1990, 9(8):235।-2359.

II. Neuwald AF, Kannan N, Poleksic A, Hata N, Liu JS: Ran's C-terminal, basic patch and nucleotide exchange mechanisms in light of a canonical structure for Rab, Rho, Ras and Ran GTPases. Genome Res 2003, 13(4):673-692.

12. Neuwald AF: The CHAIN program: forging evolutionary links to underlying mechanisms. Trends Biochem Sci 2007, 32(II):487-493.

13. Vetter IR, Wittinghofer $A:$ The guanine nucleotide-binding switch in three dimensions. Science 200I, 294(5545): I 299-1 304.

14. Weiss MS, Brandl M, Suhnel J, Pal D, Hilgenfeld R: More hydrogen bonds for the (structural) biologist. Trends Biochem Sci 200I, 26(9):52I-523.

15. Merkel JS, Regan L: Aromatic rescue of glycine in beta sheets. Fold Des 1998, 3(6):449-455.

16. Burley SK, Petsko GA: Amino-aromatic interactions in proteins. FEBS Lett 1986, 203(2):139-143.

17. Ye M, Shima F, Muraoka S, Liao J, Okamoto H, Yamamoto M, Tamura A, Yagi N, Ueki T, Kataoka T: Crystal structure of M-Ras reveals a GTP-bound "off" state conformation of Ras family small GTPases. J Biol Chem 2005, 280(35):3 |267-3I 275.

18. Eathiraj S, Pan X, Ritacco C, Lambright DG: Structural basis of family-wide Rab GTPase recognition by rabenosyn-5. Nature 2005, 436(7049):4I5-4I9.

19. Fujikawa K, Satoh AK, Kawamura S, Ozaki K: Molecular and functional characterization of a unique Rab protein, RABRPI, containing the WDIAGQE sequence in a GTPase motif. Zoolog Sci 2002, I 9(9):98I-993.

20. Renault L, Kuhlmann J, Henkel A, Wittinghofer A: Structural basis for guanine nucleotide exchange on Ran by the regulator of chromosome condensation (RCCI). Cell 200I, I 05(2):245-255.

21. Bone S: Structural flexibility in hydrated proteins. J Phys Chem B 2008, I I 2(32): | 007 I- I0075.
22. Schwarzinger $\mathrm{S}$, Wright $\mathrm{PE}$, Dyson $\mathrm{HJ}$ : Molecular hinges in protein folding: the urea-denatured state of apomyoglobin. Biochemistry 2002, 4I(42): I 268I-I 2686.

23. Callebaut I, Tasso A, Brasseur R, Burny A, Portetelle D, Mornon JP: Common prevalence of alanine and glycine in mobile reactive centre loops of serpins and viral fusion peptides: do prions possess a fusion peptide? J Comput Aided Mol Des 1994, 8(2): $|75-| 19 \mid$

24. Altschul SF, Madden TL, Schaffer AA, Zhang J, Zhang Z, Miller W, Lipman DJ: Gapped BLAST and PSI-BLAST: a new generation of protein database search programs. Nucleic Acids Res 1997, 25(I 7):3389-3402.

25. Neuwald AF, Liu JS, Lipman DJ, Lawrence CE: Extracting protein alignment models from the sequence database. Nucleic Acids Research 1997, 25(9):1665-1677.

26. Edgar RC: MUSCLE: a multiple sequence alignment method with reduced time and space complexity. BMC Bioinformatics 2004, 5(I): II3.

27. Edgar RC: MUSCLE: multiple sequence alignment with high accuracy and high throughput. Nucleic Acids Res 2004, 32(5): $1792-1797$

28. Liu JS, Neuwald AF, Lawrence CE: Bayesian models for multiple local sequence alignment and Gibbs sampling strategies. Am Stat Assoc 1995, 90(432): I I 56-I I 70.

29. Neuwald AF, Liu JS: Gapped alignment of protein sequence motifs through Monte Carlo optimization of a hidden Markov model. BMC Bioinformatics 2004, 5(I): 157.

30. Shindyalov IN, Bourne PE: Protein structure alignment by incremental combinatorial extension (CE) of the optimal path. Protein Eng 1998, II (9):739-747.

31. Neuwald AF: Bayesian shadows of molecular mechanisms cast in the light of evolution. Trends Biochem Sci 2006, 3 I (7):374-382.

32. Word JM, Lovell SC, Richardson JS, Richardson DC: Asparagine and glutamine: using hydrogen atom contacts in the choice of side-chain amide orientation. I $\mathrm{Mol}$ Biol 1999, 285(4): $1735-1747$.

33. Sayle RA, Milner-White EJ: RASMOL: biomolecular graphics for all. Trends Biochem Sci 1995, 20(9):374.

34. Stroupe C, Brunger AT: Crystal structures of a Rab protein in its inactive and active conformations. J Mol Biol 2000, 304(4):585-598.

35. Pasqualato S, Senic-Matuglia F, Renault L, Goud B, Salamero J, Cherfils J: The structural GDP/GTP cycle of RabII reveals a novel interface involved in the dynamics of recycling endosomes. $J$ Biol Chem 2004, 279( I 2): I |480- I | 488.

36. Tarricone C, Xiao B, Justin N, Walker PA, Rittinger K, Gamblin SJ, Smerdon SJ: The structural basis of Arfaptin-mediated crosstalk between Rac and Arf signalling pathways. Nature 200I, 4I I (6834):215-219.

37. Stewart M, Kent HM, McCoy A): The structure of the Q69L mutant of GDP-Ran shows a major conformational change in the switch II loop that accounts for its failure to bind nuclear transport factor 2 (NTF2). J Mol Biol 1998, 284(5): 15 I7- 1527.

Publish with BioMed Central and every scientist can read your work free of charge

"BioMed Central will be the most significant development for disseminating the results of biomedical research in our lifetime."

Sir Paul Nurse, Cancer Research UK

Your research papers will be:

- available free of charge to the entire biomedical community

- peer reviewed and published immediately upon acceptance

- cited in PubMed and archived on PubMed Central

- yours - you keep the copyright 